\title{
1 Curious people are less affected by social rejection
}

2 Taishi kawamoto ${ }^{1,2}$, Mitsuhiro Ura ${ }^{3}$, Kazuo Hiraki ${ }^{2}$

3

$4{ }^{1}$ Japan Society for the Promotion of Science, Tokyo, Japan

$5 \quad{ }^{2}$ Graduate School of Arts and Sciences, The University of Tokyo, Japan

$6{ }^{3}$ Department of Psychology, Ohtemon-Gakuin University, Japan

7 Corresponding author:

8 Taishi Kawamoto. Graduate School of Arts and Sciences, Tokyo University, 3-8-1,

9 Meguro-ku, Komaba, 153-8902, Japan

10 Telephone:

$11+81-82-424-6572$

12 Fax:

$13+81-82-424-6572$

14 e-mail: 
2 Recent studies have revealed that curiosity-seeking new information and

3 experiences-can improve psychological and social functioning. However, the

4 social nature of curiosity remains poorly understood. We tested whether

5 curious people show better psychological adaptation because (1) they have

6 less rejection sensitivity, and (2) they are less susceptible to daily social

7 rejection experiences. These two hypotheses were supported by a

8 cross-sectional study ( $N=500,20-39$ years old). We found that rejection

9 sensitivity partially mediates the relationship between curiosity and

10 psychological adaptation (life satisfaction and depression). Furthermore,

11 curiosity moderated the relationships between perceived daily social

12 rejection experiences and life satisfaction: Curious people are buffered

13 against such aversive effects, relative to less curious people. Our findings

14 suggest one possible explanation for why curious people experience better

15 psychological functioning: They appear to be less affected by social

16 rejection.

18 Keywords: Curiosity, Rejection sensitivity, Social rejection, Life satisfaction, 19 Depression. 


\section{Introduction}

2

Curiosity is an approach-oriented trait that is characterized as seeking new information and experiences, with an element of "seizing" pleasures and meanings they offer (Kashdan, 2009). Prior studies have revealed that curious people enjoy better psychological and social functioning. For instance, curious people report satisfaction with life and are less aggressive (Kashdan, DeWall, et al., 2013; Kashdan \& Steger, 2007). Curious people are also better at interacting with others (Kashdan, McKnight, Fincham, \& Rose, 2011; Kashdan \& Roberts, 2004) and show less defensive responses to existential threat (Kashdan, Afram, Brown, Birnbeck, \& Drvoshanov, 2011). One possible reason for these outcomes is that curious people allow themselves to experience both positive and negative feelings when they are exposed to conflict, surprise, and uncertain situations (Kashdan, DeWall, et al., 2013; Silvia, 2005). Here we sought to expand our understanding of the social nature of curiosity by focusing on another form of aversive conflict situation-social rejection. We tested two hypotheses: (a) that curious people would show better psychological adaptation, in part, because they are less rejection sensitive, and (b) that the adverse effects of social rejection on psychological adaptation would be weak for curious people, relative to less curious individuals. Rejection sensitivity is an avoidance-oriented trait that can be 
1 characterized as anxious expectation of and defensive reactions to social

2 rejection (Berenson et al., 2009; Downey \& Feldman, 1996; Kawamoto,

3 Nittono, \& Ura, 2015). These characteristics lead people with high rejection

4 sensitivity to experience various psychological difficulties, including greater

5 depression and aggression (Romero-Canyas, Downey, Berenson, Ayduk, \&

6 Kang, 2010). On the other hand, seeking new information and experiences

7 sometimes corresponds to social interaction. For example, curious people

8 often ask questions and take an interest in learning about social partners

9 (Kashdan, Sherman, Yarbro, \& Funder, 2013). They also perceive themselves

10 as less defensive, and show less anxiety during social interaction (Kashdan,

11 Sherman, et al., 2013; Silvia \& Kashdan, 2009). Such characteristics-less

12 defensiveness and anxiety-may help curious people to enjoy enhanced

13 psychological adaptation. Thus, we predicted that rejection sensitivity

14 mediates the relationship between curiosity and psychological adaptation.

15 In addition to the possibility that curious people would experience

16 less rejection sensitivity, they may be less susceptible to actual social

17 rejection during daily life. Social rejection is not an unusual event (Nezlek,

18 Wesselmann, \& Wheeler, 2012), and has strong deleterious effects on

19 psychological adaptation (Williams, 2009). Although few studies have

20 focused on the relationship between curiosity and social rejection, there is

21 some indirect evidence that curious people would cope better with social 
1 rejection. For example, a previous study revealed that curious people are

2 less aggressive when they experience hurt feelings (Kashdan, DeWall, et al.,

3 2013). Another study shows that curious people exhibit less defensive

4 responding following existential threat (Kashdan, Afram, et al., 2011), which

5 can be caused by social rejection (Williams, 2009). Thus, we predicted that

6 curiosity would modulate the relationship between social rejection and

7 psychological adaptation, although these relationships would be relatively

8 weak for curious people due to possible buffering effects of this trait.

9 1.1. The present study

We sought to investigate the social nature of curiosity by focusing on

11 rejection sensitivity and social rejection. More specifically, we tested

12 whether (a) rejection sensitivity would mediate the relationship between

13 curiosity and psychological adaptation, and (b) whether curiosity would

14 modulate the relationship between social rejection and psychological

15 adaptation. To this end, we conducted a cross-sectional survey, focusing on

16 life satisfaction and depression as measures of psychological adaptations.

17 2. Methods

\subsection{Participants}

A web survey was conducted by a pooling organization company

20 (Rakuten Research). A filler item (Please respond this item as " 1 ") was

21 randomly assigned during the questionnaire to ensure that participants were 
1 responding attentively and accurately. Data were collected from five hundred participants $(250$ females, $M$ age $=30.63$, Range $=20-39$ years old, $S D=$

3 5.35) who correctly respond to the filler item.

\subsection{Measures}

\subsubsection{Trait curiosity}

6 We used the Japanese version of the Curiosity and Exploration Inventory (J-CEI, Nishikawa, Yoshizu, Amemiya, \& Takayama, 2015), which is a translation of the Trait Curiosity and Exploration Inventory-2 (Kashdan et al., 2009). The J-CEI contains four items that measure stretching curiosity ( $\alpha$

$10=.89, M=2.18, S D=.95)$ and five items that measure embracing curiosity $(\alpha$

$11=.88, M=2.15, S D=.93)$. Because both subscales are highly correlated $(r$ $12=.81, p<.001)$, all item scores were averaged to provide a composite index 13 of curiosity $(\alpha=.93, M=2.17, \mathrm{SD}=.89)$.

\subsubsection{Rejection sensitivity} We used the 9-situation Adult Rejection Sensitivity Questionnaire

16 (Berenson et al., 2009) to measure rejection sensitivity. The A-RSQ was translated into Japanese and back translated by a translation agency

18 (Crimson Interactive Inc., Japan). For each situation, participants rated the level of anxiety or concern that they would experience about the outcome of the situation using a 6-point scale ranging from 1 (Very unconcerned) to 6

21 (Very concerned). Participants also rated the likelihood that the interaction 
1 partner would respond in an accepting manner, again using a 6-point scale

2 ranging from 1 (Very unlikely) to 6 (Very likely). A cross-situational total RSQ

3 score was computed by obtaining the mean score across the situations

4 described in the questionnaire $(\alpha=.87, M=12.33, S D=4.56)$, as was done

5 in previous studies (Berenson et al., 2009; Kawamoto, Nittono, et al., 2015).

$6 \quad$ 2.2.3. Social rejection and inclusion experiences

Daily social rejection and social inclusion experiences were measured

via a social exclusion experience questionnaire used in previous studies

9 (Kawamoto, Nittono, et al., 2015). This scale contains 11 items that measure social rejection experiences and six items that measure social inclusion experiences. Participants rated how often they experienced the scenarios during the past 6 months, on a scale from 1 (Not at all) to 5 (Very often). We used total item scores (social rejection: $\alpha=.86, M=16.58, S D=7.03$; social

14 inclusion: $\alpha=.84, M=15.98, S D=6.10)$.

\subsubsection{Life satisfaction}

Life satisfaction was measured using the five-item Satisfaction with

17 Life Scale (Diener \& Diener, 1995, Japanese version is available at 18 http://internal.psychology.illinois.edu/ ediener/SWLS.html). Participants reported the degree to which each statement applied to themselves, from 1 (not at all) to 7 (applies very well). The average score was used in the present study $(\alpha=.88, M=3.78, S D=1.38)$. 
$7 \quad$ 6.09).

\subsubsection{Depression}

Depression was measured using 10 items that comprise part of the Todai Health and Personality Inventory (Aoki et al., 1974), used in a previous social rejection study (Kawamoto, Nittono, et al., 2015). Participants rated their feelings on a 3-point scale that included 1 (No), 2 (Neither Yes nor No) and 3 (Yes). We calculated total scores for these items $(\alpha=91, M=19.91, S D$

\subsection{Data analysis}

All analyses controlled for possible effects of age and sex. First, we conducted a series of partial correlational analyses to confirm the relationships among variables. Next, we performed a mediation analysis to test for a possible mediating effect of rejection sensitivity on the relationships between curiosity and forms of psychological adaptation (i.e., depression and life satisfaction). We applied the bootstrapping method (1000 resamples) using PROCESS macro (Hayes, 2015). Finally, hieratical regression analysis was conducted to examine the possible moderating role of curiosity on the relationships between social rejection experiences and psychological adaptation.

\section{Results}

\subsection{Descriptive statistics}

As shown in Table 1, curiosity was negatively correlated with both 
1 rejection sensitivity and depression. In contrast, curiosity was positively

2 correlated with life satisfaction, social inclusion experiences, and social

3 rejection experiences. In addition, rejection sensitivity was positively

4 correlated with depression and negatively correlated with life satisfaction.

5 3.2. Mediating effect of rejection sensitivity on the relationships between

$6 \quad$ curiosity and psychological adaptation

This analysis revealed partial mediation effects. The total effect of

8 curiosity on life satisfaction $(b=.46, p<.001)$ was significantly reduced upon

9 inclusion of rejection sensitivity $(b=.34, p<.001 ;$ indirect effect $=.12,95 \%$

10 confident interval from .07 to .18). Similarly, the total effect of curiosity on

11 depression $(b=-1.48, p<.001)$ was significantly reduced upon inclusion of

12 this variable $(b=-.71, p=.01$; indirect effect $=-.77,95 \%$ confident interval

13 from -1.28 to -.53$)$.

14 3.3. Moderating effect of curiosity on the relationships between social

15 rejection experiences and psychological adaptations

16 Hierarchical regression analysis revealed a significant interaction

17 between curiosity and social rejection experiences (see Table 2). Simple

18 slope analysis indicated that the negative effect of social rejection on life

19 satisfaction was rather weak for curious people $(b=-.026, p=.006)$, as

20 compared to their less curious counterparts $(b=-.072, p<.001)$. Similarly,

21 the positive relationship between social rejection experiences and 
1 depression was weaker for curious people $(b=.27, p<.001)$ than for less

2 curious people $(b=.42, p<.001)$.

To test the robustness of the modulation effects of curiosity on psychological adaptation, rejection sensitivity was added to the model. The same interaction between curiosity and social rejection interaction was significant for life satisfaction $(b=.02, p=.02$, Figure 1$)$, but non-significant for depression $(b=-.05, p=.19)$.

\section{Discussion}

Curious people seek out and learn from conflict and uncertain experiences with open mind, which in turn promotes better psychological functioning (Kashdan, 2009; Silvia \& Kashdan, 2009). We sought to develop our understanding of the social nature of curiosity by examining the relationship between this trait, social rejection, and psychological adaptation. We found that rejection sensitivity partially mediates the relationships between curiosity and psychological adaptation. In addition, curiosity moderates the relationship between social rejection and life satisfaction, such that this negative relationship was weak for curious people. These findings extend curiosity research by showing that curious people seem to have advantageous characteristics related to social rejection. Prior studies have suggested that curious people cope better with conflict and uncertain situations (Kashdan, 2009; Silvia \& Kashdan, 2009). These 
1 tendencies appear to extend to social interaction, although how curiosity is

2 related to rejection sensitivity or social rejection has been largely

3 unexplored. Our findings are in line with previous studies indicating that

4 curious people are good at maintaining social interaction (Kashdan, McKnight,

5 et al., 2011; Kashdan \& Roberts, 2004) and are less aggressive when they feel

6 hurt (Kashdan, DeWall, et al., 2013).

We also found two unexpected and interesting relationships that could serve to invigorate future research on curiosity. First, although we

9 found a modulation effect of curiosity on life satisfaction, the effect on depression was not robust. This finding may be due to a particularly strong

11 effect of rejection sensitivity on depression (Romero-Canyas et al., 2010), as

12 well as a relatively specific effect of curiosity on positive psychological

13 functions (Javanovic \& Brdaric, 2012). Given that social rejection causes

14 dynamic processes in our brain, processes that impact cognition, affect, and

15 behavior (Kawamoto, Ura, \& Nittono, 2015), future studies should 16 experimentally investigate how curious people respond to social rejection.

17 Second, curiosity was positively correlated with social rejection experiences.

18 This result could be interpreted based on at least two possibilities: That 19 curious people tends to experience social rejection, or that social rejection 20 increases curiosity. Future studies would be benefit from using longitudinal 21 designs to identify the potential causal relationship between curiosity and 
1 social rejection.

2 On the basis of the above discussion, it is suggested that extreme

3 forms of curiosity might be linked to sensation seeking and risky behaviors

4 (e.g., Zuckerman, 2010). Certain aspects of sensitivity to social rejection

5 could be adaptive (e.g., Del Giudice, Ellis, \& Shirtcliff, 2011; Kawamoto,

6 Nittono, et al., 2015). Future studies focusing on sensation seeking, risky

7 behaviors, and adaptive aspects of sensitivity to social rejection are

8 expected to improve our understanding of the social nature of curiosity.

9 To conclude, we found that curious people are less rejection sensitive,

10 which in turn is associated with better psychological adaptation. Curious

11 people are also less affected by the adverse effects of social rejection on life

12 satisfaction. The present study serves to highlight the social strengths

13 enjoyed by the curious.

14 


\section{References}

Aoki, S., Suzuki, S., \& Yanai, H. (1994). A new trial of making a health and personality inventry, THPI. The Japanese Journal of Behaviometrics, 2, 41-53.

Berenson, K. R., Gyurak, A., Ayduk, O., Downey, G., Garner, M. J., Mogg, K., . . Pine, D. S. (2009). Rejection sensitivity and disruption of attention by social threat cues. Journal of Research in Personality, 43, 1064-1072. doi:10.1016/j.jrp.2009.07.007

Del Giudice, M., Ellis, B. J., \& Shirtcliff, E. A. (2011). The adaptive calibration model of stress responsivity. Neuroscience \& Biobehavioral Reviews, 35, 1562-1592. doi: 10.1016/j.neubiorev.2010.11.007

Diener, E., \& Diener, M. (1995). Cross-cultural correlates of life satisfaction and self-esteem. Journal of Personality and Social Psychology, 68, 653-663. doi:10.1037/0022-3514.68.4.653

Downey, G., \& Feldman, S. I. (1996). Implications of rejection sensitivity for intimate relationships. Journal of Personality and Social Psychology, 70, 1327-1343. doi: 10.1037/0022-3514.61.2.226

Hayes, A. F. (2015). An index and test of linear moderated mediation. Multivariate Behavioral Research, 50, 1-22. doi: $10.1080 / 00273171.2014 .962683$

Javanovic, V., \& Brdaric, D. (2012). Did curiosity kill the cat? Evidence from 
subjective well-being in adolescents. Personality and Individual Diffrences, 52, 380-384. doi:10.1016/j.paid.2011.10.043

Kashdan, T. B. (2009). Curious? Discover the missing ingredient to a fulfilling life. New York, NY: William Morrow.

Kashdan, T. B., Afram, A., Brown, K. W., Birnbeck, M., \& Drvoshanov, M. (2011). Curiosity enhances the role of mindfulness in reducing defensive responses to existential threat. Personality and Individual Differences, 50, 1227-1232. doi:1016/j.paid.2011.02.015

Kashdan, T. B., DeWall, C. N., Pond, R. S., Silvia, P. J., Lambert, N. M., Fincham, F. D., . . . Keller, P. S. (2013). Curiosity protects against interpersonal aggression: Cross-sectional, daily process, and behavioral evidence. Journal of Personality, 81, 87-102. doi:10.1111/j.1467-6494.2012.00783.x

Kashdan, T. B., Gallagher, M. W., Silvia, P. J., Winterstein, B. P., Breen, W. E., Terhar, D., \& Steger, M. F. (2009). The Curiosity and Exploration Inventory-II: Development, factor structure, and psychometrics. Journal of Research in Personality, 43, 987-998. doi:10.1016/j.jrp.2009.04.011

Kashdan, T. B., McKnight, P. E., Fincham, F. D., \& Rose, P. (2011). When curiosity breeds intimacy: Taking advantage of intimacy opportunities and transforming boring conversations. Journal of Personality, 79, 
1369-1402. doi:10.1111/j.1467-6494.2010.00697.x

Kashdan, T. B., \& Roberts, J. E. (2004). Trait and state curiosity in the genesis of intimacy: Differentiation from related constructs. Journal of Social and Clinical Psychology, 23, 792-816. doi:10.1521/jscp.23.6.792.54800

Kashdan, T. B., Sherman, R. A., Yarbro, J., \& Funder, D. C. (2013). How are curious people viewed and how do they behave in social situations? From the perspectives of self, friends, parents, and unacquainted observers. Journal of Personality, 81, 142-154. doi:10.1111/j.1467-6494.2012.00796.x

Kashdan, T. B., \& Steger, M. F. (2007). Curiosity and pathways to well-being and meaning in life: Traits, stat. Motivation and Emotion, 31, 159-173. doi:10.1007/s11031-007-9068-7

Kawamoto, T., Nittono, H., \& Ura, M. (2015). Trait rejection sensitivity is associated with vigilance and defensive response rather than detection of social rejection cues. Frontiers in Psychology, 6, 1516. doi:10.3389/fpsyg.2015.01516

Kawamoto, T., Ura, M., \& Nittono, H. (2015). Intrapersonal and interpersonal processes of social exclusion. Frontiers in Neuroscience, 9, 62. doi:10.3389/fnins.2015.00062

Nezlek, J. B., Wesselmann, E. D., \& Wheeler, L. W., K. D. (2012). Ostracism in everyday life. Group Dynamics: Theory, Research, and Practice, 16, 
Nishikawa, K., Yoshizu, J., Amemiya, T., \& Takayama, N. (2015). Individual differences in trait curiosities and their rekations to mental mealth and psychological well-being. Journal of Japan Health Medicine Association, 24, 40-48.

Romero-Canyas, R., Downey, G., Berenson, K., Ayduk, O., \& Kang, N. J. (2010). Rejection sensitivity and the rejection-hostility link in romantic relationships. Journal of Personality, 78, 119-148. doi:10.1111/j.1467-6494.2009.00611.x

Silvia, P. J. (2005). What is interesting? Exploring the appraisal structure of interest. Emotion, 5, 89-102. doi:10.1037/1528-3542.5.1.89

Silvia, P. J., \& Kashdan, T. B. (2009). Interesting things and curious people: Exploration and engagement as transient states and enduring strengths. Personality and Social Psychologi Compass, 3, 785-797. doi: 10.1111/j.1751-9004.2009.00210.x.

Williams, K. D. (2009). Ostracism: A temporal need-threat model. In M. P. Zanna (Ed.), Advanced in Experimental Social Psychology (Vol. 41, pp. 275-314). Yew York: Academic Press.

Zuckerman, M. (2010). Sensation Seeking. Corsini Encyclopedia of Psychology. 1-4. doi: 10.1002/9780470479216.corpsy0843 


\section{Acknowledgement}

2

This work was supported by CREST from the JST, Grant-in-Aid for JSPS

3 Fellows (15J07499) and for Scientific Research B (15H03449) from the Japan

4 Society for Promotion of Sciences.

5 
1 Table 1. Partial correlations among variables (controlling age and sex)

\begin{tabular}{lrrrrr}
\hline & 1 & 2 & 3 & 4 & 5 \\
\hline $\begin{array}{l}\text { 1. Curiosity } \\
\text { 2. Rejection }\end{array}$ & $-.27^{*}$ & - & & & \\
sensitivity & & & & & \\
3. Depression & $-.22^{*}$ & $.45^{*}$ & - & & \\
4. Life Satisfaction & $.30^{*}$ & $-.35^{*}$ & $-.63^{*}$ & - & \\
5. Social inclusion & $.40^{*}$ & $-.25^{*}$ & $-.33^{*}$ & $.32^{*}$ & - \\
6. Social rejection & $.26^{*}$ & $.18^{*}$ & $.21^{*}$ & -.05 & $.26^{*}$ \\
\hline
\end{tabular}

$2 \quad$ Note: $* p<.05$

3

4 
1 Table 2. Results of hierarchical regression analysis

2

\begin{tabular}{|c|c|c|c|c|c|c|}
\hline & \multicolumn{3}{|c|}{ Life satisfaction } & \multicolumn{3}{|c|}{ Depression } \\
\hline & Step1 & Step2 & Step3 & Step1 & Step2 & Step3 \\
\hline Intercept & $3.775^{*}$ & $3.775^{*}$ & $3.757^{*}$ & $19.908^{*}$ & $19.908^{*}$ & $19.782 *$ \\
\hline Sex & $.298^{*}$ & .153 & .135 & -.065 & $1.171^{*}$ & $1.263^{*}$ \\
\hline Age & -.009 & .005 & .004 & .080 & .023 & .024 \\
\hline Curiosity & & $.365 *$ & $.377^{*}$ & & $-1.151 *$ & $-1.326^{*}$ \\
\hline Social rejection & & $-.037^{*}$ & $-.049 *$ & & $.306^{*}$ & $.342 *$ \\
\hline Social inclusion & & $.063^{*}$ & $.064^{*}$ & & $-.358^{*}$ & $-.357^{*}$ \\
\hline Curiosity* & & & $.026^{*}$ & & & $-.082 *$ \\
\hline \multicolumn{7}{|l|}{ social rejection } \\
\hline Curiosity* & & & -.013 & & & $.129 *$ \\
\hline \multicolumn{7}{|l|}{ social inclusion } \\
\hline$R^{2}$ & $.013^{*}$ & $.178 *$ & $.194^{*}$ & .005 & $.228^{*}$ & $.245^{*}$ \\
\hline$\Delta R^{2}$ & & $.165^{*}$ & $.015^{*}$ & & $.223 *$ & $.017^{*}$ \\
\hline
\end{tabular}

3 Note: ${ }^{*} p<.05$

4

5 
2 The modulation effect of curiosity on the relationship between social

3 rejection experiences and life satisfaction $(* p<.001,+p=.097)$. Error bars

4 indicate standard error.

5

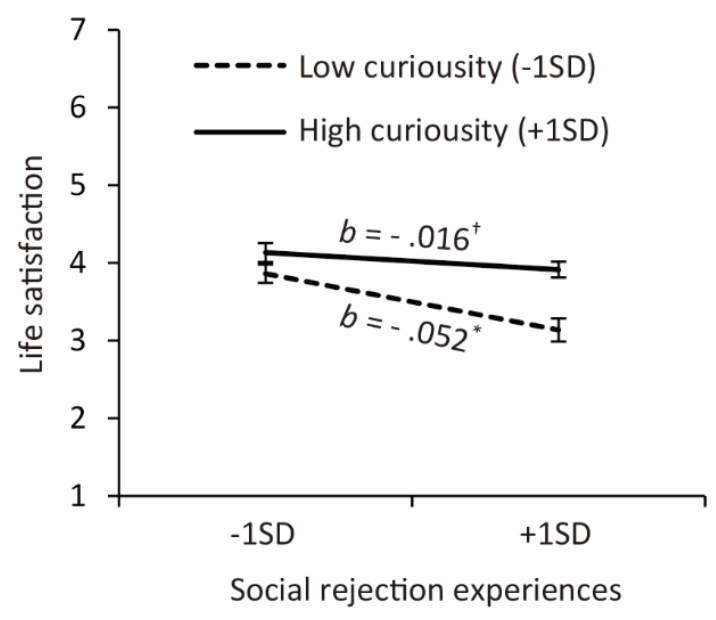

